\title{
COMPONENTES PRINCIPAIS E REGRESSÃO MÚLTIPLA NA ESTIMATIVA DA PRODUTIVIDADE DE CAFÉ COM BASE EM NUTRIENTES FOLIARES
}

\author{
Laís Barreto Franco ${ }^{1}$ \\ Jorge Tadeu Fim Rosas ${ }^{2}$ \\ Michel de Assis Silva ${ }^{3}$ \\ Julião Soares de Souza Lima ${ }^{4}$ \\ Samuel de Assis Silva ${ }^{5}$
}

Resumo: Com esse trabalho se objetivou, através de componentes principais e regressão múltipla, estimar a produtividade de café com base em nutrientes foliares. A pesquisa foi desenvolvida em uma área cultivada com café arábica - Coffea arabica L.. Construiu-se uma grade irregular com 100 pontos amostrais. Para a realização da análise foliar, foram amostradas folhas do terceiro e quarto pares do ramo produtivo. $A$ produção foi avaliada em julho de 2008 e convertida em produtividade. Os valores encontrados foram submetidos a uma análise de componentes principais (PCA). Com a finalidade de encontrar um modelo para a produtividade, foram ajustados modelos de regressão linear múltipla utilizando as componentes principais geradas. A análise geoestatística foi utilizada para quantificar o grau de dependência espacial. Comprovada a dependência, construiu-se mapas temáticos. A estimativa de produtividade a partir de modelos de regressão múltipla utilizando as componentes principais retornou valores semelhantes aos observados para os dados reais. Essa ferramenta é eficiente para estimar a produtividade com base nas componentes geradas à partir dos nutrientes foliares.

Palavras-chave: Agricultura de Precisão, estatística multivariada, regressão múltipla, cafeicultura.

\footnotetext{
${ }^{1}$ Universidade Estadual de Santa Cruz, Brasil. E-mail: laisfranco.agro@yahoo.com.br.

2 Universidade Federal do Espírito Santo, Brasil. E-mail: jorgetadeufimrosas@hotmail.com.

3 Universidade Federal do Espírito Santo, Brasil. E-mail: michelsilv@outlook.com.

${ }^{4}$ Universidade Federal do Espírito Santo, Brasil. E-mail: limajss@yahoo.com.br.

5 Universidade Federal do Espírito Santo, Brasil. E-mail: samuel.assilva@gmail.com.
} 